\title{
'|||||||||||||||||||||||||||||||||||||||||||||||||||||||||||||||||||||||.
}

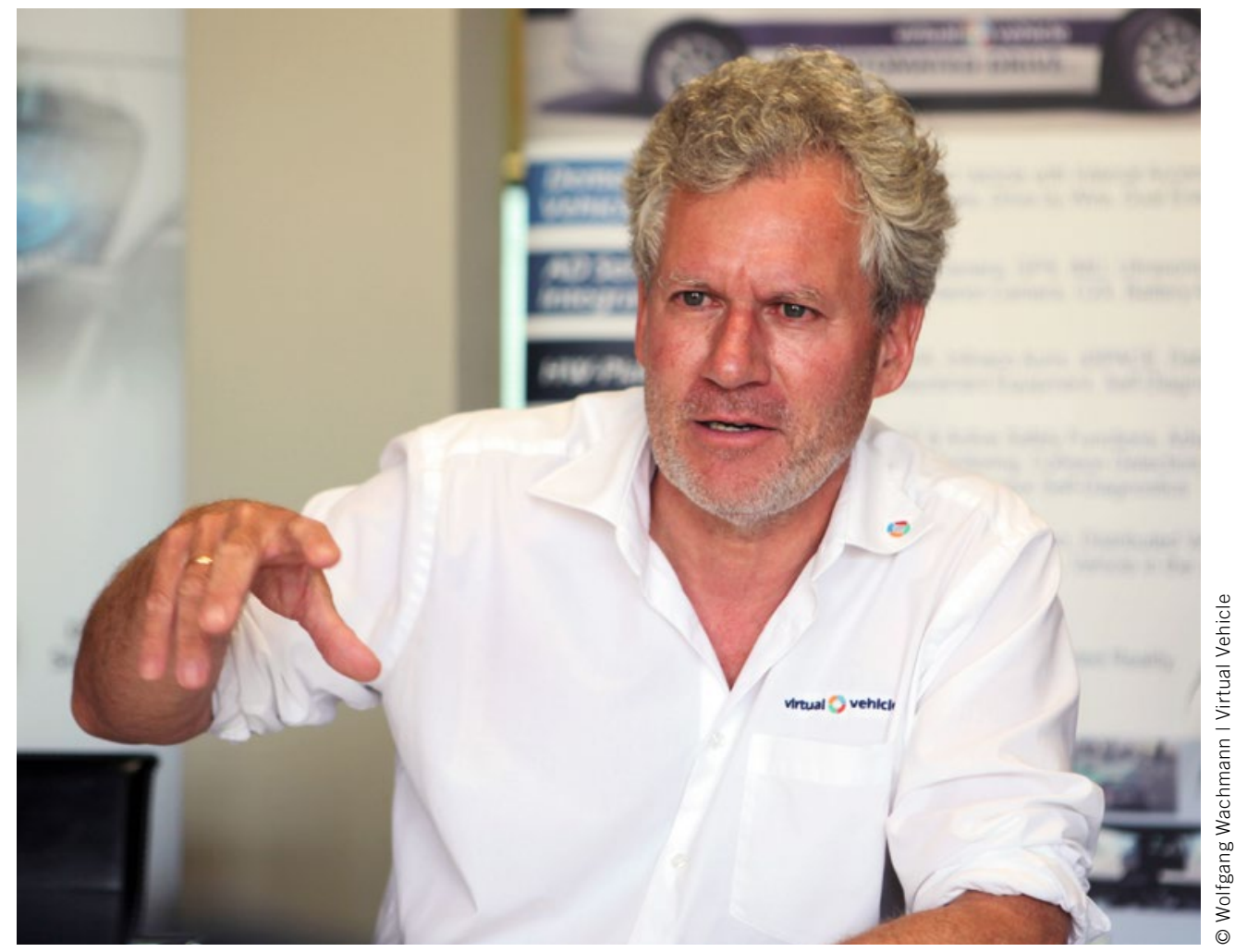

Dr. rer. nat. Jost Bernasch CEO of

Virtual Vehicle Research $\mathrm{GmbH}$

\section{The Only Rule - Software Wins}

In the race for tomorrow's mobility, it is all about one rule: Software wins. Similar to Roborace, the international racing series for automated vehicles, the path to victory is written with software. The mechanical vehicle structure becomes a matter of course, and it loses its impact as a sales argument. Styling and new functions are dominant. In times of software-defined vehicles, primarily mechanical objects are turned into globally networked, Over-the-Air (OTA) updateable high-tech driving systems as part of the internet of things - in the future called the economy of things.

Tesla managed to take the lead. But this is not a drag race which is won by a good initial acceleration. The mobility of tomorrow is a long-distance race. Everyday suitability, comfort and strategy are just as important as maintenance, customer understanding and mass production. Here, classic OEMs score points with their experience - and meanwhile all the big teams have entered this race. One thing is obvious: On the road, safety and security go beyond speed of development. New E/E architectures with central domain computers and new functional safety and security concepts make the software-based vehicles safe and suitable for everyday use. Future software architectures are the key to competition between OEMs and the new vehicle manufacturers who are just starting from scratch.
All of this requires a drastic restructuring of the development processes and more cross-domain cooperation. Complete-system thinking paired with continuous testing will shape the development of complex systems. The complete system simulation and function-oriented development are of significant importance and supported by approaches of model-based systems engineering. As realized in successfully established software projects, agile development replaces a milestone-driven "big-bang integration" through continuous integration of development results and automated tests. Virtual Vehicle Research supports several big players in strategic partnerships in the paradigm shift for these new processes, methods and technologies. Together we are working on a new generation of system simulations with trustworthy, reliable and certifiable results as credible simulation. A continuous integration of subsystems and data will be the key to innovation, development and competitiveness and will ensure future success.

The international race for the mobility of the future has only just begun, but every meter of lead that is now achieved will be crucial. Because one aspect of this race must be considered: There is no finish line - instead, participants who are too slow are mercilessly overtaken, and consequently out of the race. 